\title{
Adaptação e Análise da Estrutura Interna da Escala de Mobbing de Leymann (LIPT45) para o Contexto Português
}

\author{
Cristiana Máximo, José Soares Martins \\ Universidade Fernando Pessoa, Porto, Portugal \\ Sergio Dominguez-Lara ${ }^{1}$ \\ Universidad de San Martín de Porres, Lima, Peru \\ Abílio Lourenço \\ CIPE - Centro de Investigação em Psicologia e Educação da Escola Secundária Alexandre Herculano \\ Margarida Simões \\ Universidade de Trás-os-Montes e Alto Douro, Vila Real, Portugal
}

RESUMO

O objetivo deste trabalho consiste em adaptar e analisar a estrutura interna da versão portuguesa do Leymann Inventory of Psychological Terror (LIPT45). Foi utilizada uma amostra de 404 indivíduos (70,5\% mulheres) entre os 18 e os 69 anos $(M=32,9 ; D P=12.606)$. O LIPT45-PV é uma escala de autorrelato composta por 45 itens que avaliam o assédio moral nas organizações, divididos em cinco dimensões: efeitos na autoexpressão; efeitos sobre os contatos sociais; efeitos sobre a reputação pessoal; efeitos sobre a situação ocupacional e qualidade de vida, e efeitos sobre a saúde. Foi utilizada uma análise fatorial confirmatória e o Exploratory Structural Equation Modeling (ESEM), mediante os quais se avaliou um modelo de cinco dimensões, além da confiabilidade do construto e das pontuações. Em conformidade com os resultados da ESEM, o LIPT45-PV apresenta uma estrutura fatorial coerente, assim como uma maior diferenciação entre as suas dimensões. Da mesma forma, os indicadores de confiabilidade do construto e das pontuações foram adequados. Conclui-se que o LIPT45-PV revela características psicométricas (estrutura interna e confiabilidade) que o configura como um instrumento adequado para avaliar o respetivo constructo em adultos.

Palavras-chave: adaptação; mobbing; maltrato laboral; abuso emocional.

ABSTRACT - Adaptation and analysis of the internal structure of the Leymann Inventory of Psychological Terror (LIPT45) for the Portuguese context

The aim of this work was to adapt and analyze the internal structure of the Portuguese version of the Leymann Inventory of Psychological Terror (LIPT45). A sample of 404 individuals (70.5\% women) aged between 18 and 69 years $(M=32.9 ; S D=12.606)$ was used. The LIPT45-PV is a self-report scale composed by 45 items that assess mobbing in organizations, divided into five dimensions: effects on self-expression; effects on social contacts; effects on personal reputation; effects on the occupational situation and quality of life, and effects on health. Confirmatory factor analysis and Exploratory Structural Equation Modeling (ESEM) were used, through which a five-dimensional model was evaluated, as well as the reliability of the construct and the scores. According to the results of the ESEM, the LIPT45-PV presents a coherent factor structure, as well as greater differentiation between its dimensions. Likewise, the reliability indicators of the construct and the results were adequate. We can conclude that the LIPT45-PV reveals psychometric characteristics (internal structure and reliability) that configure it as an adequate instrument to evaluate the respective construct in adults.

Keywords: Adaptation; Mobbing; Mistreatment at Work; Emotional Abuse.

RESUMEN - Adaptación y análisis de la estructura interna de la escala de mobbing de Leymann (LIPT45) para el contexto portugués

El objetivo de este trabajo consiste en adaptar y analizar la estructura interna de la versión portuguesa del Leymann Inventory of Psychological Terror (LIPT45). Fue utilizada una muestra de 404 individuos (70.5\% mujeres) entre los 18 y 69 años $(M=32.9$; $D S=12.606)$. El LIPT45-PV es una escala de autoinforme compuesta por 45 ítems que evalúan el acoso laboral en las organizaciones, divididos en cinco dimensiones: efectos en la autoexpresión; efectos sobre los contactos sociales; efectos sobre la reputación personal; efectos sobre la situación ocupacional y calidad de vida, y efectos sobre la salud. Fue utilizado un análisis factorial confirmatorio y el Exploratory Structural Equation Modeling (ESEM), a través de los cuales se evaluó un modelo de cinco dimensiones, además de la confiabilidad del constructo y de las puntuaciones. De acuerdo con los resultados del ESEM, el LIPT45-PV presenta una estructura factorial coherente, así como una mayor diferenciación entre sus dimensiones. Asimismo, los indicadores de confiabilidad del constructo y de las puntuaciones fueron adecuados. Se concluye que el LIPT45-PV presenta características psicométricas (estructura interna y confiabilidad) que lo configuran como un instrumento adecuado para evaluar el constructo mencionado en adultos. Palabras clave: Adaptación; Mobbing; Acoso Laboral; Abuso Emocional. 
Em Portugal, não são conhecidas estatísticas atuais, mas estima-se que haja uma alta ocorrência de assédio moral e psicológico no local de trabalho, tendo como consequência o absentismo e uma série de problemas psicossociais, assim como não existem instrumentos fiáveis e validados que avaliem adequadamente esse fenômeno no nosso contexto sociocultural (Villar, Caputo, Coria, \& Messoulam, 2012). Numa perspetiva jurídico-legal, o assédio só passou a ser legislado no Código do Trabalho (CT) a partir de 2003, com a introdução da Lei 99/2003, de 27 de agosto, estando inserido no artigo $24^{\circ}$. Com a última atualização desse mesmo código, datado de outubro de 2017, o assédio passou a ter enquadramento legal através do artigo $29^{\circ}$.

A nível internacional, o primeiro autor que apresentou o termo "assédio moral" ou "mobbing" foi o etólogo Konnrad Lorenz, em 1963, para descrever o comportamento animal, referindo-se ao ataque ameaçador ou comportamento de um grupo de animais menores a um animal maior (Leymann, 1996; Pascu, 2015; Pereira, 2009). Posteriormente, o médico sueco Heinemann, em 1972, utilizou o termo "mobbing", anteriormente referido por Lorenz, para descrever os comportamentos disruptivos de pequenos grupos de crianças contra uma única criança, durante o período de aulas (Leymann, 1996; Pereira, 2009).

Na sequência desses estudos, surge a obra da autoria da psiquiatra Carroll M. Brodsky, intitulada The Harassed Work, no ano de 1976, em que fala sobre as relações violentas surgidas nos locais de trabalho. No ano de 1993, o psiquiatra alemão Heinz Leymann, publicou o seu estudo - Mobbing -, acerca da perseguição no trabalho e, depois a psiquiatra francesa Marie-France Hiriegoyen (2001), publicou um trabalho reconhecido como Le Harcèlement moral la violence perverse au quotidien (Pereira, 2009). Contudo, os primeiros estudos científicos sobre o fenômeno de mobbing tiveram a sua origem na Europa, mais concretamente na Suécia, com Leymann, seguido por vários autores em diferentes países. Assim, o mobbing é considerado um dos efeitos das novas políticas de gestão, resultado das imposições do capitalismo neoliberal, da competição, da globalização e das relações interpessoais contraditórias, cuja predominância advém dos países de maior poder econômico e político a nível mundial (Saraiva \& Pinto, 2011).

De acordo com Leymann (1990, 1996), o conceito de mobbing significa assediar ou aterrorizar psicologicamente outras pessoas no local de trabalho, referindo que esse fenômeno é bastante antigo, contudo apenas começou a ser descrito e investigado, de uma forma mais sistemática, a partir da década de 80 . Nessa sequência, Leymann (1990) enumerou quatro fases: a de conflito ou acontecimento crítico original; de mobbing e estigmatização; de administração de pessoal ou de intervenção na empresa; e, por último, de expulsão ou marginalização da vida laboral. O autor indica, igualmente, que essa situação social não é linear, isto é, não existem graus de mobbing - ou se é vítima ou não é.

Leymann, em 1989, começa a desenvolver o questionário LIPT (Leymann Inventory of Psychological Terrorization), para estabelecer a frequência de mobbing numa organização. Dessa forma, o método foi validado e conseguiu diferenciar entre funcionários com "mobbed" e "não mobbed". Esse questionário é composto por 45 itens agrupados por cinco dimensões, designadamente: efeitos na autoexpressão; efeitos sobre os contatos sociais; efeitos sobre a reputação social; efeitos sobre a situação ocupacional e qualidade de vida e efeitos sobre a saúde. Como se pode observar, os nomes das dimensões são autoexplicativos e refletem o impacto em diferentes esferas da vida do trabalhador.

No ano de 2005, González de Rivera e RodríguezAbuín adaptaram o (LIPT) para Espanha, onde adicionaram 15 comportamentos e avaliaram a frequência e a intensidade dos comportamentos compreendidos pelo entrevistado, dando assim origem ao Cuestionario de Estratégias de Acosso en el Trabajo - o LIPT-60 modificado, tendo os autores encontrado seis dimensões. O acréscimo desses quinze itens, que acrescenta uma nova dimensão na escala espanhola, é justificada pelos autores como tendo sido detetadas, desde o início das investigações em Espanha, estratégias de assédio que não estavam contempladas na escala original de Leymann (1990).

Em Portugal, recorrendo aos estudos desenvolvidos por González de Rivera e Rodríguez-Abuín (2005), surgem os estudos no sentido de colmatar uma lacuna investigativa sentida nessa área, assim como devido a um incremento de interesse nessa problemática, em várias ambiências laborais.

No entanto, os estudos realizados têm algumas limitações que impedem o seu uso em contextos aplicados. Em primeiro lugar, aparece o estudo de Carvalho (2007) em que os profissionais de enfermagem, apesar dos objetivos de validação da escala, não apresentam os procedimentos e os resultados relacionados com esta. Posteriormente, Carvalho (2010) e João (2012) apresentaram propostas estruturais alternativas do LIPT, mas ao fundamentar a análise num procedimento questionável como o Little Jiffy (Lloret-Segura, Ferreres-Traver, Hernández-Baeza, \& Thomas-Marco de 2014), baseando-se em componentes principais e eigenvalues (valores próprios) por meio de uma rotação varimax, os resultados são questionáveis. Nesse sentido, é necessário implementar estudos analíticos fatoriais com o rigor dos desenvolvimentos mais recentes, como o Exploratory Structural Equation Modeling (ESEM; Asparouhov \& Muthén, 2009), cuja aplicação resultou num ajuste de estimativa mais precisa dos dados e correlações interfatoriais (Marsh, Morin, Parker, \& Kaur, 2014) em instrumentos multifatoriais. Além disso, esse método não foi aplicado ao LIPT, por isso representam uma novidade na análise estrutural do instrumento. 
Dada a pouca pesquisa realizada em Portugal, o reduzido número de estudos científicos que analisem as questões de assédio moral no local de trabalho, considerou-se ser pertinente e adequado a adaptação e análise da estrutura interna do LIPT45 original, ao contexto português. Devido à projeção internacional que a escala inicial de Leymann (1990) assume no contexto organizacional, foi opção manter os mesmos 45 itens distribuídos pelas mesmas cinco dimensões, passando esta nova escala a ser designada por Leymann Inventory of Psychological Terrorization - Portuguese Version (LIPT45-PV).

Assim, este estudo está norteado para adaptar e fazer a análise da estrutura interna da escala original de Leymann (1990) - LIPT45 -, de forma a que se possam identificar e avaliar as diferentes situações/estratégias de assédio moral, físico e psicológico que ocorrem no espaço laboral, nomeadamente as que produzem um descrédito ou desprestígio no trabalho, bem como aquelas que, por meio de distorção de comunicação (rumores, calúnias), com medidas restritivas ou de comparação pejorativa com os restantes trabalhadores, minimizam ou prejudicam as suas realizações.

\section{Método}

\section{Participantes}

Foi utilizada uma amostra de 404 indivíduos, de algumas instituições da zona norte do país. Elas foram escolhidas por motivos de ordem pragmática - nomeadamente de caráter geográfico - não havendo, dessa forma, qualquer presunção de generalizar os resultados obtidos à população portuguesa. A amostra era constituída por $119(29,5 \%)$ sujeitos do género masculino e $285(70,5 \%)$ do feminino, com idades compreendidas entre os $18 \mathrm{e}$ os 69 anos cuja média de idade para a amostra total é de 32,9 anos $(D P=12,61)$, sendo de $32,5(D P=12,14)$ anos para o gênero masculino e de $33,1(D P=12,80)$ para o feminino.

\section{Instrumento}

Leymann Inventory of Psychological TerrorPortuguese Version (LIPT45-PV) é um instrumento que apresenta 45 itens com cinco opções de resposta: Nada em absoluto (0); Um pouco (1); Moderadamente (2); Muito (3); e Extremamente (4). Os itens estão distribuídos por cinco dimensões: Efeitos da Autoexpressão (EAE), os comportamentos incluídos nessa dimensão sugerem comportamentos que têm como objetivo bloquear a comunicação tanto dentro como fora da empresa (e.g., "Os seus superiores não o deixam expressar-se ou dizer o que tem para dizer") corresponde à soma das pontuações de onze itens: a dimensão Efeitos nos Contatos Sociais (ECS), Os comportamentos vão no sentido de não dirigir a palavra ao trabalhador, garantido que ninguém o faz, cortando-se as suas fontes de informação e isolando-a fisicamente dos seus colegas (e.g., "Não consegue falar com ninguém, todos os evitam") é medida por meio de cinco itens; a dimensão dos Efeitos na Reputação Social (ERS), os comportamentos incluídos nessa dimensão têm como objetivo o desmérito do trabalhador, usando a distorção da comunicação, murmúrios, difamações e isolamento (e.g., "Fazem circular boatos falsos ou sem fundamento sobre sî") é identificado por quinze itens; a dimensão que respeita aos Efeitos na Situação Ocupacional e Qualidade de Vida (ESOQV), os comportamentos incluídos nessa dimensão sugerem comportamentos de rebaixamento do trabalhador com tarefas impróprias para as suas capacidades e danos dissimulados para que não seja possível identificar os responsáveis (e.g., "Obrigam-no a realizar tarefas absurdas ou inúteis") é alcançada a partir de sete itens; e, finalmente, a dimensão relativa aos Efeitos na Saúde (ES), os comportamentos visam comprometer a saúde do trabalhador, como: ataques diretos, a mera sujeição a um regime de assédio moral com efeitos negativos, psicológicos e psicossomáticos (e.g., "Obrigam-no a realizar trabalhos nocivos ou perigosos") é estabelecida tendo em conta o somatório das pontuações em sete itens.

\section{Procedimentos}

O LIPT-45 foi traduzido e submetido a uma retroversão por dois especialistas da língua inglesa, um dos quais nativo do mesmo idioma e, posteriormente, sujeito a uma triagem por dois psicólogos. Nesse seguimento, foram realizados pré-testes em amostras equivalentes à atual, cujos resultados serviram para aprimorar os inventários, tendo por base a compreensão semântica dos itens.

Posteriormente, com o objetivo de obter autorização superior para a aplicação do questionário foram contatadas as respetivas direções das instituições, onde foi explicada a finalidade do estudo. O LIPT45-PV foi administrado em contexto laboral, durante o ano de 2017. O procedimento de recolha de dados ocorreu de forma diferenciada, tendo-se tido em atenção a especificidade da instituição e respetivas rotinas e o tempo de aplicação foi em média 20 a 30 minutos. Na maioria dos casos foi possível aplicar o questionário com a presença do investigador, tendo sido solicitado aos inquiridos que respondessem com o máximo de sinceridade, tentando não esquecer nenhum item. Noutros casos, os questionários foram deixados no órgão de direção da instituição e recolhidos posteriormente. Os professores foram bastante recetivos, tendo sido garantidos os procedimentos éticos e deontológicos convencionais, designadamente a confidencialidade das respostas e a voluntariedade na participação. Apenas participaram os docentes que, anteriormente, assinaram o consentimento informado, descrito na declaração de Helsínquia para estudos com humanos.

\section{Análise de Dados}

Análise preliminar. Relativamente à análise preliminar, foi examinada a distribuição das variáveis (assimetrias e curtoses) e, desse modo, verificar se um conjunto 
de dados podem ter sido gerados a partir de uma distribuição normal. Considerando o critério de Finney e DiStefano (2013), em que 2 e 7 são identificados como valores máximos admissíveis para assimetria e curtose, respetivamente.

Análise estrutural. $\mathrm{O}$ modelo de cinco dimensões do LIPT-45 foi avaliado segundo duas perspetivas. A primeira foi a análise confirmatória (AFC), mediante o método de estimação dos mínimos quadrados ponderados com variância ajustada (WLSMV) com matrizes policóricas, considerando o caráter ordinal dos itens, assim como o valor da assimetria e curtose de alguns itens e a preferência generalizada dos respondentes pelas opções mais baixas na escala de respostas. De forma complementar, foram analisadas as más especificações ( $\mathrm{Hu} \&$ Bentler, 1998), associadas a potenciais cargas cruzadas ou residuais correlacionadas no modelo, com base na informação dada pelos índices de modificação (IM; Sörbom, 1989), sob a abordagem de Saris, Satora e Van der Veld (2009) usando um módulo especializado (DominguezLara, 2018a). A segunda aproximação realizada mediante o Exploratory Structural Equation Modeling (ESEM; Asparouhov \& Muthén, 2009) com o método de rotação geomin (Asparouhov \& Muthén, 2009), o qual estima livremente as cargas fatoriais dos itens em todos os fatores.

A avaliação de cada modelo foi feita por vários índices de ajuste, tais como o RMSEA (<.06) (Kline, 2010), CFI $(\geq 0,95)$ (Kline, 2010) e o WRMR (<0,90; Yu, 2002), embora os pontos de corte sejam aproximações, uma vez que alguns índices de ajustamento podem ser alterados por características inerentes ao modelo (e.g., Kenny, Kaniskan, \& McCoach, 2015). As cargas fatoriais de cada um dos modelos foram avaliados segundo o seu valor, esperando que eles sejam maiores que 0,60 (Dominguez-Lara, 2018b). Para os procedimentos descritos anteriormente, foi utilizado o programa Mplus, versão 7 (Muthen \& Muthen, 1998-2015).

Com ambos os modelos, foi analisada a validade convergente e discriminante em cada uma das dimensões. Para a validade interna convergente, foi calculada a variância média extraída (Average Variance Extracted AVE), que representa o grau em que os itens são explicados pela variável latente, considerando-se aceitáveis valores próximos de 0,50 (Fornell \& Larcker, 1981). A validade interna discriminante comprova se a variância partilhada entre fatores (correlação interfatorial ao quadrado; $\varphi^{2}$ ) é menor que o AVE de cada fator envolvido na correlação, a fim de se concluir sobre a ausência de sobreposição ou diferenciação empírica entre constructos. A Figura 1 representa a especificação pictórica do modelo de cinco dimensões do LIPT45-PV.

Confiabilidade. Com o melhor modelo foi analisada a confiabilidade do construto mediante o coeficiente $\omega$ (McDonald, 1999) e $H$ (Hancock \& Mueller, 2001) em que os valores maiores que 0,80 são considerados adequados (Raykov \& Hancock, 2005). Por último, foram calculados os coeficientes $\alpha$ com intervalos de confiança (IC; Dominguez-Lara, 2016).

De uma forma complementar, foi analisada a confiabilidade da diferença entre escores $(\rho d)$ no interior do perfil configurado pelas dimensões do LIPT-45 (Muñiz, 2003; Dominguez-Lara, 2018c). O pd pode estar localizado entre zero e a unidade, e o seu valor indica o grau em que a diferença entre resultados tem mais a ver com a variância verdadeira do que com a variância do erro. Nesse caso, se $\rho d$ possui valores adequados (e.g., >0,70), o perfil é aceitavelmente confiável, ou seja, a sua configuração fornece informações relevantes sobre as dimensões do mobbing avaliadas.

\section{Resultados}

\section{Análise Descritiva}

Relativamente à análise descritiva realizada, pode observar-se (c.f. Tabela 1) que os indicadores de assimetria e curtose estão em conformidade com o critério de Finney e DiStefano (2013).

\section{Estrutura Interna}

A avaliação do modelo de cinco dimensões representativo do LIPT45-PV evidenciou índices de ajustamento adequados. Em primeiro lugar, para o modelo sob o CFA, esses índices estiveram dentro do aceitável (CFI =0,94; RMSEA [IC 90\%]=0,03 [0,02, 0,03]; WRMR=0,99). Além disso, de acordo com o IM, havia catorze cargas cruzadas potencialmente significativas, das quais cinco podem qualificar-se como más especificações, ou seja, que a sua modelação é necessária para melhorar o fator de estrutura, além de dois residuais correlacionados. Essa constatação justifica a implementação do ESEM. Quanto a avaliação do modelo sob a abordagem ESEM, os indicadores foram melhores que o CFA (CFI =0,98; RMSEA $[\mathrm{IC} 90 \%]=0,02[0,01 ; .0,02]$; WRMR $=0,64)$.

As cargas fatoriais foram heterogêneas no interior de cada fator (Tabela 2), chegando inclusive a valores que poderiam ser considerados baixos $(<0,30)$, pelo qual o AVE de diversos fatores poderiam ser considerados baixos $(<0,40)$. No caso do ESEM, apenas dois itens tiveram cargas fatoriais significativas num fator o que não era esperado (carga cruzada) (Tabela 2).

Por último, relativamente às correlações interfatoriais, essas foram estatisticamente significativas, e não sugerem multicolinearidade em ambos os modelos $(<0,80)$, apesar de o modelo ESEM melhorar o discriminante de validade interna, ou seja, que a variância compartilhada $\left(\varphi^{2}\right)$ é menor do que o AVE (Tabela 2).

Em termos gerais, as cargas fatoriais sob a abordagem CFA foram aceitáveis na maioria dos casos $(\approx 0,50)$ e, embora não tenham diminuído significativamente, ao estimar as cargas cruzadas sob a metodologia ESEM, as novas cargas fatoriais indicam que a representatividade de cada constructo é baixa em alguns itens $(\approx 0,40)$. Por 
outro lado, havia alguns itens complexos, como o item 23 (Imitam a sua voz, a sua maneira de andar os seus gestos para fazer pouco de si) originalmente de "efeitos na reputação social", que também representa "Efeitos nos
Contactos Sociais"; e o item 26 (Gozam com sua nacionalidade, a sua naturalidade ou a sua origem) de "efeitos na saúde" que representa melhor o construto "efeitos na reputação social".

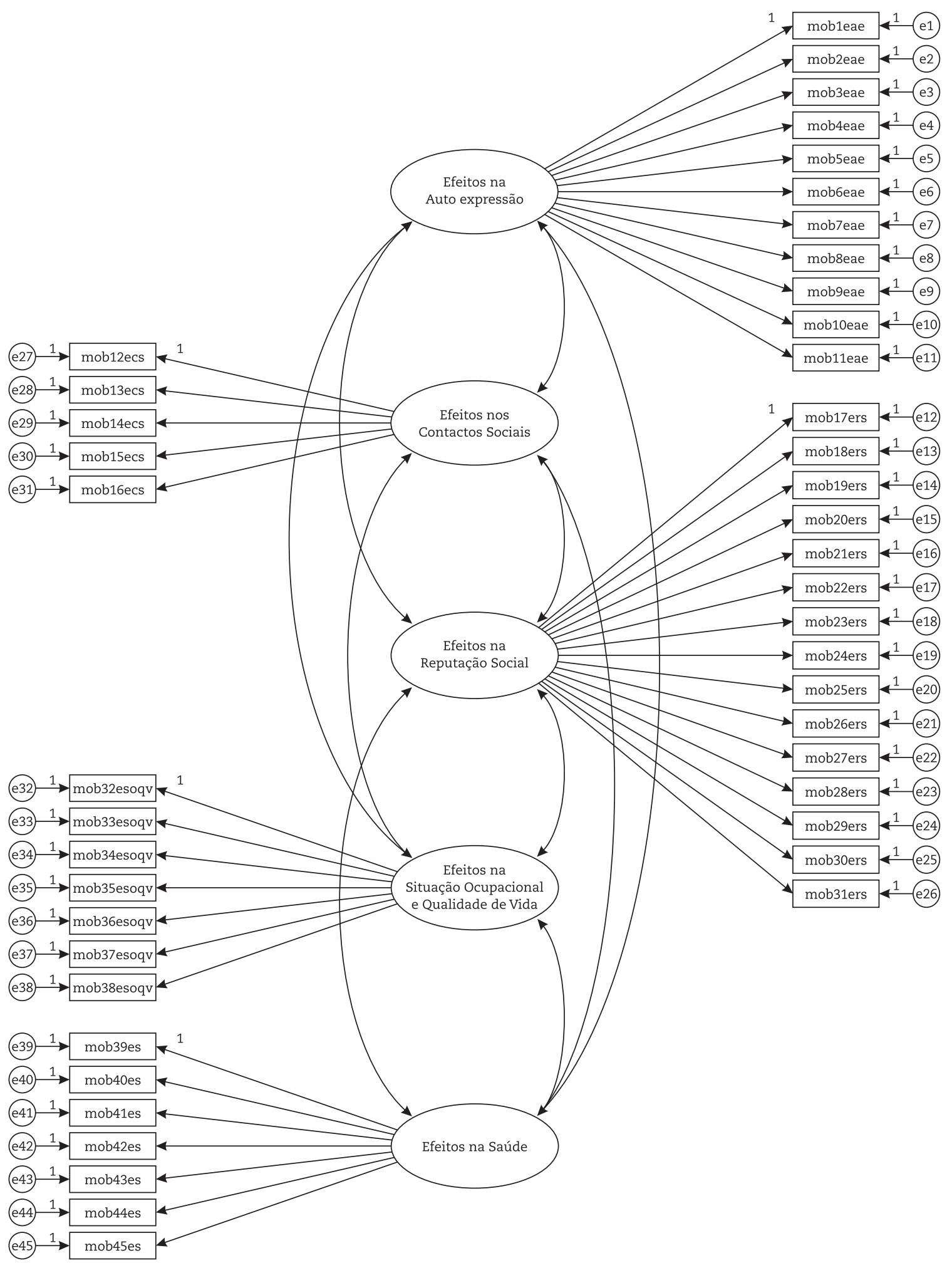

Figura 1. Especificação pictórica do modelo de cinco dimensões do LIPT45-PV 
Tabela 1

Análise Descritiva dos Itens do LIPT45-PV

\begin{tabular}{|c|c|c|c|c|}
\hline Ítens & Média & $\mathrm{DP}$ & Assimetria & Curtose \\
\hline Íten 39-ES & 0,84 & 1,28 & 1,27 & 0,24 \\
\hline Íten 45-ES & 0,71 & 1,08 & 1,39 & 0,92 \\
\hline Íten 44-ES & 0,87 & 1,26 & 1,23 & 0,255 \\
\hline Íten 43-ES & 0,80 & 1,07 & 1,17 & 0,41 \\
\hline Íten 42-ES & 0,74 & 1,06 & 1,32 & 0,83 \\
\hline Íten 41-ES & 0,81 & 1,22 & 1,39 & 0,74 \\
\hline Íten 40-ES & 0,84 & 1,26 & 1,22 & 0,16 \\
\hline Íten 38-ESOQV & 0,78 & 1,22 & 1,38 & 0,66 \\
\hline Íten 37-ESOQV & 0,76 & 1,15 & 1,42 & 0,99 \\
\hline Íten 36-ESOQV & 0,79 & 1,14 & 1,32 & 0,68 \\
\hline Íten 35-ESOQV & 0,80 & 1,16 & 1,31 & 0,67 \\
\hline Íten 34-ESOQV & 0,84 & 1,22 & 1,31 & 0,59 \\
\hline Íten 33-ESOQV & 1,01 & 1,28 & 1,11 & 0,10 \\
\hline Íten 32-ESOQV & 0,88 & 1,25 & 1,18 & 0,20 \\
\hline Íten 29-ERS & 0,95 & 1,33 & 1,24 & 0,23 \\
\hline Íten 31-ERS & 0,84 & 1,2 & 1,27 & 0,49 \\
\hline Íten 30-ERS & 0,84 & 1,19 & 1,35 & 0,74 \\
\hline Íten 28-ERS & 0,98 & 1,41 & 1,22 & 0,02 \\
\hline Íten 25-ERS & 0,80 & 1,26 & 1,47 & 0,90 \\
\hline Íten 17-ERS & 0,93 & 1,38 & 1,29 & 0,16 \\
\hline Íten 27-ERS & 0,86 & 1,26 & 1,36 & 0,65 \\
\hline Íten 26-ERS & 0,84 & 1,26 & 1,39 & 0,69 \\
\hline Íten 19-ERS & 0,85 & 1,23 & 1,36 & 0,74 \\
\hline Íten 18-ERS & 0,93 & 1,42 & 1,28 & 0,07 \\
\hline Íten 21-ERS & 0,96 & 1,39 & 1,21 & 0,02 \\
\hline Íten 20-ERS & 0,78 & 1,19 & 1,34 & 0,57 \\
\hline Íten 23-ERS & 0,88 & 1,34 & 1,31 & 0,32 \\
\hline Íten 22-ERS & 0,78 & 1,21 & 1,35 & 0,55 \\
\hline Íten 24-ERS & 0,85 & 1,31 & 1,34 & 0,40 \\
\hline Íten 16-ECS & 0,81 & 1,25 & 1,28 & 0,24 \\
\hline Íten 15-ECS & 0,77 & 1,24 & 1,34 & 0,37 \\
\hline Íten 14-ECS & 0,83 & 1,26 & 1,21 & 0,06 \\
\hline Íten 13-ECS & 0,78 & 1,2 & 1,3 & 0,40 \\
\hline Íten 12-ECS & 0,99 & 1,33 & 1,2 & 0,14 \\
\hline Íten 1-EAE & 1,14 & 1,22 & 1,01 & 0,04 \\
\hline Íten 9-EAE & 0,84 & 1,18 & 1,37 & 0,94 \\
\hline Íten 11-EAE & 1,03 & 1,3 & 1,11 & 0,05 \\
\hline Íten 10-EAE & 0,78 & 1,12 & 1,38 & 0,98 \\
\hline Íten 3-EAE & 0,90 & 1,19 & 1,36 & 0,94 \\
\hline Íten 2-EAE & 1,08 & 1,2 & 1 & 0,05 \\
\hline Íten 5-EAE & 0,92 & 1,1 & 1,23 & 0,83 \\
\hline Íten 4-EAE & 0,88 & 1,18 & 1,29 & 0,68 \\
\hline Íten 7-EAE & 0,81 & 1,23 & 1,33 & 0,54 \\
\hline Íten 6-EAE & 0,86 & 1,22 & 1,4 & 0,93 \\
\hline Íten 8-EAE & 0,80 & 1,25 & 1,34 & 0,45 \\
\hline
\end{tabular}

Nota. DP=Desvio padrão; ES=Efeitos na saúde; ESOQV=Efeitos na situação ocupacional e qualidade de vida; ERS=Efeitos na reputação social; ECS=Efeitos nos contactos sociais; EAE=Efeitos da autoexpressão 
Tabela 2

Estrutura Fatorial y Parâmetros Descritivos do Inventário LIPT45-PV

\begin{tabular}{|c|c|c|c|c|c|c|c|c|c|c|c|c|}
\hline \multirow{2}{*}{ Itens } & \multicolumn{6}{|c|}{ CFA } & \multicolumn{6}{|c|}{ ESEM } \\
\hline & F1 & F2 & F3 & F4 & F5 & $h^{2}$ & F1 & F2 & F3 & F4 & F5 & $\mathrm{h}^{2}$ \\
\hline Íten 1 & 0,44 & & & & & 0,19 & 0,41 & 0,08 & $-0,02$ & 0,11 & 0,02 & 0,17 \\
\hline Íten 2 & 0,65 & & & & & 0,42 & 0,50 & 0,05 & 0,12 & 0,15 & 0,17 & 0,25 \\
\hline Íten 3 & 0,62 & & & & & 0,39 & 0,50 & 0,13 & 0,05 & 0,17 & 0,09 & 0,25 \\
\hline Íten 4 & 0,69 & & & & & 0,48 & 0,61 & 0,08 & 0,02 & 0,16 & 0,09 & 0,37 \\
\hline Íten 5 & 0,64 & & & & & 0,42 & 0,62 & $-0,11$ & 0,00 & 0,17 & 0,16 & 0,38 \\
\hline Íten 6 & 0,73 & & & & & 0,53 & 0,58 & 0,14 & 0,17 & 0,11 & 0,13 & 0,34 \\
\hline Íten 7 & 0,52 & & & & & 0,27 & 0,50 & $-0,04$ & $-0,05$ & 0,07 & 0,2 & 0,25 \\
\hline Íten 8 & 0,59 & & & & & 0,35 & 0,58 & 0,16 & $-0,08$ & $-0,07$ & 0,25 & 0,33 \\
\hline Íten 9 & 0,51 & & & & & 0,26 & 0,52 & $-0,09$ & $-0,02$ & 0,08 & 0,16 & 0,28 \\
\hline Íten 10 & 0,65 & & & & & 0,42 & 0,56 & 0,03 & 0,08 & 0,12 & 0,15 & 0,31 \\
\hline Íten 11 & 0,54 & & & & & 0,30 & 0,53 & $-0,10$ & $-0,04$ & 0,13 & 0,16 & 0,28 \\
\hline Íten 12 & & 0,61 & & & & 0,38 & 0,00 & 0,70 & $-0,15$ & 0,05 & 0,06 & 0,48 \\
\hline Íten 13 & & 0,67 & & & & 0,45 & $-0,02$ & 0,70 & $-0,14$ & 0,13 & 0,02 & 0,49 \\
\hline Íten 14 & & 0,81 & & & & 0,65 & $-0,09$ & 0,62 & 0,06 & 0,30 & 0,03 & 0,39 \\
\hline Íten 15 & & 0,87 & & & & 0,76 & $-0,04$ & 0,75 & 0,05 & 0,22 & 0,02 & 0,57 \\
\hline Íten 16 & & 0,78 & & & & 0,60 & 0,07 & 0,73 & $-0,01$ & 0,11 & 0,06 & 0,54 \\
\hline Íten 17 & & & 0,63 & & & 0,39 & 0,08 & 0,18 & 0,42 & 0,13 & 0,19 & 0,18 \\
\hline Íten 18 & & & 0,48 & & & 0,23 & 0,04 & 0,11 & 0,41 & 0,01 & 0,21 & 0,17 \\
\hline Íten 19 & & & 0,51 & & & 0,26 & 0,06 & $-0,02$ & 0,52 & 0,10 & 0,12 & 0,27 \\
\hline Íten 20 & & & 0,63 & & & 0,40 & 0,06 & 0,15 & 0,55 & 0,16 & $-0,01$ & 0,30 \\
\hline Íten 21 & & & 0,42 & & & 0,18 & 0,02 & 0,19 & 0,43 & $-0,05$ & $-0,10$ & 0,18 \\
\hline Íten 22 & & & 0,59 & & & 0,34 & $-0,12$ & 0,21 & 0,57 & 0,06 & $-0,08$ & 0,32 \\
\hline Íten 23 & & & 0,65 & & & 0,43 & 0,03 & 0,44 & 0,35 & $-0,02$ & 0,09 & 0,12 \\
\hline Íten 24 & & & 0,62 & & & 0,38 & $-0,07$ & 0,15 & 0,56 & 0,17 & $-0,07$ & 0,31 \\
\hline Íten 25 & & & 0,52 & & & 0,27 & 0,03 & 0,24 & 0,43 & $-0,07$ & 0,09 & 0,19 \\
\hline Íten 26 & & & 0,23 & & & 0,05 & 0,03 & $-0,17$ & 0,27 & 0,05 & 0,33 & 0,07 \\
\hline Íten 27 & & & 0,53 & & & 0,29 & 0,05 & 0,19 & 0,41 & 0,02 & 0,15 & 0,17 \\
\hline Íten 28 & & & 0,53 & & & 0,28 & $-0,12$ & 0,08 & 0,46 & 0,17 & 0,13 & 0,21 \\
\hline Íten 29 & & & 0,51 & & & 0,26 & 0,03 & 0,08 & 0,48 & 0,15 & $-0,08$ & 0,23 \\
\hline Íten 30 & & & 0,53 & & & 0,28 & 0,04 & 0,07 & 0,45 & 0,12 & 0,12 & 0,20 \\
\hline Íten 31 & & & 0,47 & & & 0,22 & 0,11 & 0,15 & 0,40 & $-0,05$ & 0,10 & 0,16 \\
\hline Íten 32 & & & & 0,58 & & 0,33 & $-0,03$ & $-0,03$ & $-0,02$ & 0,57 & 0,14 & 0,32 \\
\hline Íten 33 & & & & 0,48 & & 0,23 & 0,01 & 0,10 & $-0,09$ & 0,47 & $-0,02$ & 0,23 \\
\hline Íten 34 & & & & 0,73 & & 0,53 & $-0,01$ & 0,07 & $-0,01$ & 0,65 & 0,10 & 0,43 \\
\hline Íten 35 & & & & 0,61 & & 0,38 & $-0,04$ & $-0,11$ & $-0,11$ & 0,74 & 0,10 & 0,54 \\
\hline Íten 36 & & & & 0,67 & & 0,45 & $-0,11$ & 0,10 & 0,00 & 0,63 & 0,02 & 0,40 \\
\hline Íten 37 & & & & 0,62 & & 0,38 & $-0,09$ & 0,15 & $-0,09$ & 0,55 & 0,08 & 0,30 \\
\hline Íten 38 & & & & 0,63 & & 0,39 & $-0,14$ & 0,12 & 0,05 & 0,54 & 0,08 & 0,29 \\
\hline Íten 39 & & & & & 0,55 & 0,31 & $-0,05$ & $-0,07$ & $-0,15$ & 0,04 & 0,52 & 0,27 \\
\hline Íten 40 & & & & & 0,38 & 0,14 & $-0,12$ & $-0,02$ & 0,01 & 0,03 & 0,34 & 0,12 \\
\hline Íten 41 & & & & & 0,45 & 0,21 & $-0,11$ & 0,00 & $-0,05$ & $-0,09$ & 0,48 & 0,23 \\
\hline Íten 42 & & & & & 0,55 & 0,30 & $-0,19$ & 0,04 & $-0,09$ & $-0,03$ & 0,54 & 0,29 \\
\hline Íten 43 & & & & & 0,66 & 0,43 & $-0,24$ & $-0,12$ & 0,02 & $-0,03$ & 0,70 & 0,49 \\
\hline Íten 44 & & & & & 0,60 & 0,36 & $-0,11$ & $-0,03$ & $-0,11$ & 0,00 & 0,58 & 0,34 \\
\hline Íten 45 & & & & & 0,66 & 0,44 & $-0,11$ & 0,08 & $-0,13$ & $-00,1$ & 0,66 & 0,44 \\
\hline
\end{tabular}


Tabela 2 (continuação)

Estrutura Fatorial y Parâmetros Descritivos do Inventário LIPT45-PV

\begin{tabular}{|c|c|c|c|c|c|c|c|c|c|c|c|c|}
\hline \multirow{2}{*}{ Itens } & \multicolumn{6}{|c|}{ CFA } & \multicolumn{6}{|c|}{ ESEM } \\
\hline & F1 & F2 & F3 & F4 & F5 & $h^{2}$ & F1 & F2 & F3 & F4 & F5 & $h^{2}$ \\
\hline AVE & 0,37 & 0,57 & 0,28 & 0,38 & 0,31 & & 0,29 & 0,49 & 0,20 & 0,36 & 0,31 & \\
\hline F1 & 1 & 0,05 & 0,08 & 0,09 & 0,01 & & 1 & 0,00 & 0,00 & 0,01 & 0,01 & \\
\hline F2 & 0,22 & 1 & 0,32 & 0,30 & 0,00 & & 0,04 & 1 & 0,07 & 0,08 & 0,00 & \\
\hline F3 & 0,27 & 0,57 & 1 & 0,11 & 0,00 & & 0,06 & 0,26 & 1 & 0,01 & 0,00 & \\
\hline F4 & 0,29 & 0,55 & 0,33 & 1 & 0,03 & & 0,12 & 0,28 & 0,12 & 1 & 0,02 & \\
\hline F5 & 0,11 & 0,04 & 0,03 & 0,17 & 1 & & 0,11 & 0,06 & 0,05 & 0,15 & 1 & \\
\hline Média & 10,03 & 4,17 & 13,05 & 5,86 & 5,61 & & - & - & - & - & - & \\
\hline DP & 7,93 & 4,69 & 10,15 & 5,30 & 4,93 & & - & - & - & - & - & \\
\hline
\end{tabular}

Nota. N=404; F1=efeitos da autoexpressão; F2=Efeitos nos Contactos Sociais; F3=efeitos na reputação social; F4=efeitos na situação ocupacional e qualidade de vida; $F 5=$ efeitos na saúde; $h^{2}=$ Comunalidade; sublinhado=Cargas fatoriais acima da 0,30 em outro fator; Abaixo da diagonal=correlações interfatoriais; Acima da diagonal=Variância partilhada entre fatores $\left(\phi^{2}\right)$; IC=intervalo de confiança; DP=Desvio padrão

\section{Confiabilidade}

Em termos gerais, a confiabilidade do constructo, tanto em $\omega$ como em $H$ alcançaram valores adequados na maioria dos casos $(\geq 0,80)$ : efeitos da autoexpressão $(\omega=0,82 ; H=0,82)$, efeitos nos contactos sociais $(\omega=0,83$; $H=0,83)$, efeitos na reputação social $(\omega=0,79 ; H=0,80)$, efeitos na situação ocupacional e qualidade de vida $(\omega=0,793$; $H=0,808)$, e efeitos na saúde $(\omega=0,75 ; H=0,78)$.

Relativamente à confiabilidade das pontuações, os coeficientes $\alpha$ mostraram valores aceitáveis (limite inferior do IC $>0,70$, na maioria dos casos) para os objetivos da investigação: efeitos da autoexpressão $(\alpha=0,82$; IC95\% $0,79-0,85$ ), efeitos nos contatos sociais ( $\alpha=0,80$; IC95\% $0,77-0,84)$, efeitos na reputação social $(\alpha=0,81$; IC95\% 0,77 - 0,84), efeitos na situação ocupacional e qualidade de vida ( $\alpha=0,75$; IC95\% 0,70 - 0,79), e efeitos na saúde ( $\alpha=0,70$; IC95\% 0,65-0,75).

Finalmente, em relação à confiabilidade das diferenças, os valores de $\rho d$ eram aceitáveis $(\rho d$-média $=0,73)$ e apenas a diferença entre duas pontuações (efeitos nos contatos sociais e efeitos na situação ocupacional e qualidade de vida) obteve um valor abaixo das expectativas $(\rho d=0,64)$.

\section{Discussão}

Da revisão da literatura constata-se que são escassas as investigações que se debrucem sobre o estudo das circunstâncias de assédio moral e psicológico nos distintos locais de trabalho, tendo como resultado o absentismo e os consequentes dilemas psicossociais que daí advêm, mais especificamente quando se pretende avaliar os efeitos provocados nos indivíduos, em contexto laboral, ao nível das autoexpressões, dos contatos sociais, da reputação social, da situação ocupacional e qualidade de vida, bem com na saúde física e psicológica dos envolvidos.
Tendo como suporte o trabalho iniciado por Leymann (1990), foram desenvolvidos outros estudos sobre o mobbing (Carvalho, 2007, 2010; Gonzalez de Rivera \& Rodríguez-Abuin, 2005; João, 2012; Luna, 2003), embora tenham sofrido algumas alterações ao nível do número das dimensões tratadas, geralmente baseados em procedimentos não recomendados. $\mathrm{O}$ trabalho de Gonzalez de Rivera e Rodríguez-Abuin (2005) reporta uma primeira adaptação e validação do LIPT45 para o contexto espanhol e é apresentado com seis subescalas de assédio, num total de sessenta itens. A escala, inicialmente de tipo dicotômico, é alterada para uma métrica de resposta do tipo Likert, devido à necessidade sentida pelos autores de valorizar a intensidade com que os inquiridos são afetados por cada uma das situações/estratégias de assédio. Essa nova escala (LIPT-60) apresenta, nas suas seis dimensões, valores de alfa de Cronbach entre 0,83 e 0,94.

No estudo de Carvalho (2007) a autora, por meio de uma amostra de conveniência constituída por setenta enfermeiros portugueses e utilizando o LIPT-60 (Gonzalez de Rivera \& Rodríguez-Abuin, 2005), refere que a maioria dos inquiridos experienciou no seu local de trabalho, pelo menos, uma das sessenta situações de assédio moral, físico ou psicológico apresentado no questionário. Realça condutas, tais como - "interrompem-no quando fala", criticam o seu trabalho", "caluniam-no e falam nas suas costas", "criticam a sua vida privada" e "gritam-lhe ou repreendem-no em voz alta" -, que o agressor utiliza como método para desconsiderar a vítima perante os seus colegas de trabalho, assim como limitar a sua comunicação. O estudo refere, ainda, que é evidente o impedimento à comunicação e existe uma pressão frequente em invadir a vida particular e profissional da vítima. Contudo, não é apresentado no estudo qualquer tipo de valores da estrutura interna da escala, pelo que é impossível fazer o contraste com as conclusões da presente investigação. 
Noutro estudo de João (2012), a autora utilizou igualmente o LIPT-60 (Gonzalez de Rivera \& RodríguezAbuin, 2005) com uma amostra de 3227 enfermeiros de Portugal continental, Açores e Madeira e, embora os resultados tenham como principal limitação o método utilizado, a sua conclusão é relevante - o mobbing como uma realidade tangível em muitos locais de trabalho e que lhe estão inerentes consequências não só para a vítima, assim como para a própria organização e sociedade em geral. A autora menciona, ainda, ser de importância fundamental continuar a detectar eventuais vítimas e agressores nos contextos organizacionais, a fim de se poder atuar em tempo útil na minimização ou eliminação de danos psicológicos e físicos.

De realçar que o LIPT45-PV, além de facultar uma informação global - frequência e intensidade percebida do conjunto de comportamentos e estratégias de assédio sofridas pelos indivíduos -, permite, igualmente, dar a conhecer mais especificamente a tipologia comportamental desse mesmo assédio moral, físico ou psicológico. Das análises descritivas obtidas, é possível verificar, em cada uma das cinco dimensões, que constituem a escala, quais as condutas mais referidas pelos respondentes. Assim, quando nos referimos aos "Efeitos da Autoexpressão", os indivíduos mencionam que os seus superiores não os deixam expressar-se ou dizerem o que têm para dizer e ignoram a sua presença, não respondendo ás suas perguntas e, quanto aos "Efeitos nos Contactos Sociais", afirmam que os colegas de trabalho deixaram ou estão a deixar de dirigir-se a si ou de falar consigo, como também lhe designam um local de trabalho que o mantém isolado dos restantes colegas. Relativamente aos "Efeitos na Reputação Social", os inquiridos mencionam que avaliam o seu trabalho de maneira parcial e mal-intencionada e que os colegas de trabalho querem obrigá-lo (a) a que faça um exame psiquiátrico ou psicológico. No respeitante aos "Efeitos na Situação Ocupacional e Qualidade de Vida", atitudes como cortarem as suas iniciativas e não lhe permitirem desenvolver as suas ideias, assim como não lhe determinarem novas tarefas ou algo para fazer, são as condutas mais frequentes de assédio moral, físico ou psicológico no trabalho. Finalmente, no que concerne aos "Efeitos na Saúde", os sujeitos da amostra referem que provocam danos no seu domicílio e no seu posto de trabalho e obrigam-no a realizar trabalhos nocivos ou perigosos. Por outro lado, os comportamentos de assédio menos mencionados pelos inquiridos da amostra, referem-se a serem vítimas de agressões físicas e sexuais diretas, obrigarem a realizar tarefas humilhantes, proibirem os seus colegas de falarem consigo, não olharem para si ou olharem-no com desprezo e gestos de desdém e tratarem-no como se fosse um doente mental ou darem-no a entender. Embora a relevância concedida aos comportamentos associados aos distintos assédios laborais seja relativamente recente, as constatações dos resultados dos estudos realizados no seu âmbito, realçam a importância do seu impacto na vida das empresas, mais especificamente na dos indivíduos que lá trabalham.

Por essa razão, o LIPT45-PV aduz um contributo fundamental, ao avaliar as opiniões e atitudes manifestadas pelos inquiridos no local de trabalho. Essa importância torna-se mais evidente se observarmos que a escala de Leymann (1990) está a ser adaptada e validada para o contexto português a partir da sua versão original, tendo em conta o impacto e realce que ela tem a nível internacional. Comparando o aspeto similar dos resultados obtidos com os outros estudos de validação apresentados, poder-se-á inferir que o LIPT45-PV revela características psicométricas que o configura como um instrumento fiável para avaliar o respetivo construto, podendo, seguramente, ser usado em investigações futuras, constituindo-se como uma poderosa ferramenta de intervenção organizacional.

A estrutura fatorial encontrada neste estudo, replica as escalas originais, na medida em que apresentam as mesmas dimensões (efeitos na autoexpressão, efeitos nos contactos sociais, efeitos na reputação social, efeitos na situação ocupacional e qualidade de vida, efeitos na saúde), além de apresentarem valores fatoriais robustos, que avaliam de forma adequada o construto, salientando também a diferenciação entre as dimensões. Além disso, os indicadores de confiabilidade do construto, as pontuações e diferenças entre as pontuações foram aceitáveis. Este último ponto é relevante porque, na ausência de um indicador geral de mobbing, a configuração de um perfil será a chave no momento de caracterizar os trabalhadores nas avaliações individuais e, como tal, é necessário contar com um sinalizador que avalie a sua confiabilidade.

No entanto, um aspeto não foi avaliado: a diferença mínima confiável (Charter, 1996; Dominguez-Lara, Merino-Soto, \& Navarro-Loli, 2016), ou seja, a diferença mínima esperada entre duas pontuações que podem ser consideradas confiáveis; por não ter uma amostra representativa, a estimativa desse indicador seria pouco precisa, embora seja aconselhável no futuro a realização de estudos para obter dados normativos (escalas).

Finalmente, muitas cargas fatoriais mostraram valores relativamente baixos, nessa sequência, seria aconselhável obter uma versão mais curta do LIPT-45 (e.g., quatro itens por dimensão), a fim de capturar com mais precisão o construto, além de permitir a possibilidade de avaliar mais construtos relacionados e não prolongando o tempo de aplicação, evitando, assim, o desgaste do inquirido durante o processo.

A primeira dificuldade identificada prendeu-se com o fato de a amostra circunscrever-se à zona norte do país com instituições públicas, carecendo de ser alargada numérica e geograficamente a diferentes tipos de organizações, a fim de poderem ser observadas eventuais dissemelhanças comportamentais e atitudinais dos trabalhadores, diversificando, dessa forma, os possíveis grupos de intervenção. 
Outro aspeto a considerar, diz respeito à sugestão invocada por Tashakkori e Teddlie (1998) acerca da triangulação dos dados a partir de diferentes procedimentos de avaliação. Nesse caso específico, seria interessante construir medidas de avaliação comportamental a partir de itens de questionários que possam medir esse construto como uma atitude, mas igualmente por meio de indicadores de comportamentos avaliados como um evento. O recurso a uma análise fatorial confirmatória desses dados possibilitaria identificar as comunalidades do construto medido a partir de distintas fontes de informação. Também a realização de uma entrevista individual ou uma atitude metodológica mais etnográfica no processo de recolha de dados poder-nos-ia ter conduzido a um conhecimento dos inquiridos em si mesmos.

O desenho de recolha de informação do presente estudo, é transversal, o qual não permite inferir causalidade, inclusive na perspetiva SEM. Em estudos futuros, e com o objetivo de obter informação tanto de causas e efeitos, como de possíveis relações recíprocas entre os itens (ou entre as dimensões) do instrumento, o desenho de recolha de dados deveria ser de dois ou mais momentos temporais (desenho não experimental de medidas repetidas). Um desenho desse tipo seria excelente para comprovar se existe ao longo do tempo uma interpretação coerente de opiniões e atitudes relativamente a alguns aspetos relacionados com as condutas de assédio em contexto laboral. Adicionalmente, é ainda necessário realizar estudos de invariância longitudinal, a fim de garantir que a avaliação do construto se mantenha estável. Da mesma forma, recomenda-se continuar os estudos de validação usando outras estratégias (AERA, APA, \& NCME, 2014), tais como a relação com outras variáveis ou processos de resposta, dado que obter uma evidência de prova (no caso, a estrutura interna) seja importante, não é definitiva.

Finalmente, é desejável que a investigação, nessa área ainda pouco explorada, desenvolva-se de uma forma mais profícua, por meio de um trabalho conjunto envolvendo os investigadores, as empresas públicas e privadas e demais organizações que se dedicam à implementação e desenvolvimento de programas no âmbito dos comportamentos organizacionais construtivos e de cidadania organizacional. Essas parcerias, efetivas e com continuidade no tempo, contribuirão para uma compreensão mais global e exata da problemática em estudo e a elaboração de programas de intervenção adequados. A sucessiva utilização do LIPT45-PV na investigação científica, facultará uma vasta e diversificada recolha de dados facilitadora da compreensão, não só do funcionamento desse instrumento em populações diferenciadas, assim como dos processos que concorrem para a redução de condutas desviantes em contexto laboral.

De acordo com proferido por Leymann (1996), apesar de ser manifesto algum progresso no estudo e interesse desta problemática, torna-se evidente também que ficam em aberto mais questões do que respostas empiricamente fundamentadas. Fica a expectativa de que esse quesito vai estimular mais estudos nessa área e, igualmente, consciencializar investigadores e profissionais do prejuízo e sofrimento causado pelo assédio moral, físico e psicológico em contexto laboral.

\section{Referências}

American Educational Research Association, American Psychological Association, National Council on Measurement in Education [AERA/ APA/NCME] (2014). Standards for educational and psychological testing. Washington, DC: American Psychological Association.

Asparouhov, T., \& Muthén, B. (2009). Exploratory structural equation modeling. Structural Equation Modeling, 16(3), 397-438. doi: 10.1080/10705510903008204

Brodsky, C. M. (1976). The harassed worker. Toronto, Ontario, Canada: Lexington Books, DC Heath.

Carvalho, G. (2007). Mobbing: assédio moral em contexto de enfermagem - estudo preliminar. Revista Investigação em Enfermagem, 15, 43-55. Recuperado de http://hdl.handle.net/10198/5331

Carvalho, G. (2010). Mobbing: Assédio moral em contexto de enfermagem. Revista de Investigação em Enfermagem, 21, 28-42. Recuperado de http://hdl.handle.net/10198/5327

Charter, A. R. (1996). Formulas for reliable and abnormal differences in raw test scores. Perceptual and Motor Skills, 83(3), 1017-1018. doi: 10.2466/pms.1996.83.3.1017

Dominguez-Lara, S. (2016). Intervalos de confianza en el reporte de la fiabilidad: Un análisis necesario. Anales del Sistema Sanitario de Navarra, 39(1), 169-170. doi: 10.4321/S1137-6627/2016000100024

Dominguez-Lara, S. (2018a). Procrastinación en universitarios: Evidencia preliminar del efecto de la contextualización en asignaturas específicas. Avaliação Psicológica, 17(1), 12-19. doi: 10.15689/ap.2017.1701.02.13028

Dominguez-Lara, S. (2018b). Propuesta de puntos de corte para cargas factoriales: Una perspectiva de fiabilidad de constructo. Enfermería Clínica, 28(6), 401-402. doi: 10.1016/j.enfcli.2018.06.002

Dominguez-Lara, S. (2018c). Reporte de las diferencias confiables en el perfil del ACE-III. Neurología, 33(2), 138-139. doi: 10.1016/j. nrl.2016.02.022

Dominguez-Lara, S., Merino-Soto, C., \& Navarro-Loli, J. (2016). Estimación paramétrica de la confiabilidad y diferencias confiables. Revista Médica de Chile, 144(3), 305-306. doi: 10.4067/S0034-98872016000300019

Finney, S., \& DiStefano, C. (2013). Nonnormal and categorical data in structural equation models. Em G. R. Hancock \& R. O. Mueller (Ed.), A second course in structural equation modeling (pp. 439-492). Publisher: Information Age. 
Fornell, C., \& Larcker, D. F. (1981). Evaluating structural equation models with unobservable variables and measurement error. Journal of Marketing Research, 18(1), 39-50. doi: 10.2307/3151312

González de Rivera, J. L., \& Rodríguez-Abuín, M. J. (2005). Cuestionario de estrategias de acoso en el trabajo. LIPT-60. Madrid: EOS. Em J. L. González de Rivera (Ed.), Las Claves del Mobbing. Madrid: Editorial EOS.

Hancock, G. R., \& Mueller, R. O. (2001). Rethinking construct reliability within latent variable systems. Em R. Cudeck, S. H. C. du Toit \& D. Sörbom (Eds.), Structural equation modeling: Past and present. A Festschrift in honor of Karl G. Jöreskog (pp. 195-261). Chicago: Scientific Software International.

Hirigoyen, M. F. (2001). El maltrato psicológico en la vida cotidiana. Madrid: Paidós.

Hu, L., \& Bentler, P.M. (1998). Fit indices in covariance structure modeling: Sensitivity to underparameterized model specification. Psychological Methods, 3(4), 424-453. doi: 10.1037/1082-989X.3.4.424

João, A. (2012), Estudo de validação da escala lipt-60 nos enfermeiros portugueses. International Journal of Developmental and Educational Psychology. INFAD Revista de Psicologia, 1(4), 335-343. Recuperado de http://dehesa.unex.es/handle/10662/3054

Kenny, D. A., Kaniskan, B., \& McCoach, D. B. (2015). The performance of RMSEA in models with small degrees of freedom. Sociological Methods \& Research, 44(3), 486-507. doi: 10.1177/0049124114543236

Kline, R. B. (2010). Principles and practice of structural equation modeling (3rd ed.). New York, NY: Guilford Press

Leymann, H. (1990). Mobbing and Psychological Terror at Workplaces. Violence and Victims, 5(2), 119-126. doi: 10.1891/0886-6708.5.2.119

Leymann, H. (1993). Mobbing. Psychoterror am Arbeitsplatz und wie man sich dagegen wehren kann [Mobbing. Psychological terror at the workplace and how one can defend oneself]. Reinbek bei Hamburg, Germany: Rowohlt Taschenbuch Verlag.

Leymann, H. (1996). The Content and Development of Mobbing at Work. European Journal of work and organizational psychology, 5(2), 165184. doi: 10.1080/13594329608414853

Lloret-Segura, S., Ferreres-Traver, A., Hernández-Baeza, A., \& Tomás-Marco, I. (2014). El análisis factorial exploratorio de los ítems: Una guía práctica, revisada y actualizada. Anales de Psicología, 30(3), 1151-1169. doi: 10.6018/analesps.30.3.199361

Luna, M. (2003). Acoso Psicológicoen el Trabajo (Mobbing). Ediciones Gps: Madrid.

McDonald, R. P. (1999). Test theory: A unified treatment. Mahwah, N. J.: L. Erlbaum Associates.

Marsh, H. W., Morin, A. J., Parker, P. D., \& Kaur, G. (2014). Exploratory structural equation modeling: An integration of the best features of exploratory and confirmatory factor analysis. Annual Review of Clinical Psychology, 10, 85-110. doi: 10.1146/annurevclinpsy-032813-153700

Muñiz, J. (2003). Teoría clásica de los tests. Madrid: Pirámide.

Muthén, L. K., \& Muthén, B. O. (1998-2015). Mplus User's guide (7th ed.). Los Angeles, CA: Muthén \& Muthén.

Pascu, R. (2015). The Phenomenon of Mobbing. Research and Science Today, 1(9), 146-154. Recuperado de https://heinonline.org/HOL/ LandingPage?handle $=$ hein.journals/rescito9\&div $=22 \&$ id $=\&$ page $=$

Pereira, R. (2009). Mobbing ou assédio moral no trabalho: contributos para a sua conceptualização. Coimbra: Coimbra Editora.

Raykov, T., \& Hancock, G. R. (2005). Examining change in maximal reliability for multiple-component measuring instruments. British Journal of Mathematical and Statistical Psychology, 58(1), 65-82. doi: 10.1348/000711005X38753

Tashakkori, A., \& Teddlie, C. (1998). Mixed methodology: Combining qualitative and quantitative approaches. Thousand Oaks, CA: Sage Publications Saraiva, D., \& Pinto, A. (2011). Mobbing em contexto de enfermagem. Revista de Enfermagem, 3(5), 83-93. doi: 10.12707/RIII1039

Saris, W. E., Satorra, A., \& van der Veld, W. M. (2009). Testing structural equation modeling or detection of misspecifications? Structural Equation Modeling, 16(4), 561-582. doi: 10.1080/10705510903203433

Sörbom, D. (1989). Model modification. Psychometrika, 54(3), 371-384. doi: 10.1007/BF02294623

Villar, E., Caputo, M., Coria, S., \& Messoulam, N. (2012). Psychological/Moral Workplace Harassment: Development of na Inventory in Argentina. Social Medicine, 6(4), 241-245.

Yu, C. Y. (2002). Evaluating Cut off Criteria of Model fit Indices for Latent Variable Models with Binary and Continuous Outcomes (Tese de Doutorado). Los Angeles, CA: University of California Los Angeles.

\section{Sobre os autores}

Cristiana Máximo é Licenciada em Psicologia e Mestre em Psicologia da Justiça pela Universidade Fernando Pessoa (Portugal). Estágio profissional na Segurança Social.

José Soares Martins é Licenciado em Psicologia pela Universidade do Porto e doutor em Psicologia Social pela Universidade de Santiago de Compostela. Membro no centro de Investigação em Ciências Socias da Universidade Fernando Pessoa (Portugal).

Sergio Dominguez-Lara é Psicólogo y Doutor em Psicologia. Professor do Instituto de Investigación de Psicología da Universidad de San Martín de Porres (Perú)

Abílio Lourenço é Doutor e pós-doutorado em Psicologia da Educação. Investigador sénior da Universidade do Minho, Braga (Portugal).

Margarida Simões é Doutorada em Psicologia pela Universidade da Beira Interior. Professora Auxiliar na Universidade de Trás-os-Montes e Alto Douro (Portugal). 